\title{
Estudio Sobre La Relación Entre Mobbing Y La Satisfacción Laboral En Docentes De Instituciones De Educación Superior En México
}

\author{
Juana Patricia Muñoz Chávez, Candidata PhD \\ Estudiante del Doctorado en Planeación Estratégica y Dirección de \\ Tecnología Universidad Popular Autónoma del Estado de Puebla (UPAEP), \\ México \\ Asdrúbal López-Chau, PhD \\ Profesor Investigador de Tiempo Completo, \\ Universidad Autónoma del Estado de México, CU UAEM Zumpango, \\ Miembro del Sistema Nacional de Investigación Nivel 1, México \\ Manuel González Pérez, PhD \\ Profesor Investigador de Tiempo Completo, \\ Universidad Popular Autónoma del Estado de Puebla (UPAEP), \\ Miembro del Sistema Nacional de Investigación Nivel 1, México
}

Doi: 10.19044/esj.2018.v14n4p298 URL:http://dx.doi.org/10.19044/esj.2018.v14n4p298

\begin{abstract}
Currently, the technological and administrative modernity has revolutionized the dynamics of work in organizations. In recent years, the existence of mobbing has been pointed out, which represents an obstacle to the integral development of workers; therefore, job satisfaction plays a fundamental role in the quality of life of people. In the present work a quantitative research was carried out, a transversal-correlational design was used, to determine if there is a statistically significant relationship between the mobbing variables and job satisfaction of the teachers of the Higher Education Institutions (HEI). The sample consisted of 248 teachers from four HEI in central Mexico. The hypotheses were evaluated through the chi-square statistical test. The results reveal the dependence between the dimensions of the variables analyzed.
\end{abstract}

Keywords: Mobbing, Job Satisfaction, Higher Education Institutions

\section{Resumen}

Actualmente la modernidad tecnológica y administrativa ha revolucionado las dinámicas de trabajo en las organizaciones. En los últimos años se ha puntualizado la existencia del mobbing, que representa un obstáculo 
para el desarrollo integral de los trabajadores; por ello, la satisfacción laboral juega un papel fundamental en la calidad de vida de las personas. En el presente trabajo se realizó una investigación de corte cuantitativo, se utilizó un diseño transversal-correlacional, con el objetivo de determinar si existe una relación estadísticamente significativa entre las variables mobbing y satisfacción laboral de los docentes de las Instuticiones de Educación Superior (IES). La muestra estuvo conformada por 248 docentes de cuatro IES del centro de México. Se evaluaron las hipótesis a través de la prueba estadística chi-cuadrada. Los resultados revelan la dependencia entre las dimensiones de las variables analizadas.

Palabras clave: Mobbing, Satisfacción Laboral, Instituciones de Educación Superor

\section{Introducción}

Hoy en día la forma de trabajar al interior y exterior de las organizaciones se ha visto influenciada por la globalización, lo cual, las lleva a enfrentar diversas problemáticas, por lo que, en el ámbito de los estudios organizacionales tomar conciencia de que la empresa es un ente que trabaja con personas, donde los estilos de liderazgo y el ambiente laboral juegan un papel clave en su desarrollo es fundamental. En este sentido, las organizaciones buscan un mejoramiento continuo que las lleve a elevar su productividad, y para lograrlo es importante considerar al elemento humano, el cual necesita desenvolverse en un ambiente laboral armónico que les permita desarrollarse y trabajar bien (Varela, Puhl \& Izcurdia, 2013).

Derivado de lo anterior, en la gestión organizacional la satisfacción laboral es una variable fundamental (Rodríguez, Retamal, Lizana \& Cornejo, 2011); y, el mobbing como fenómeno presente en las organizaciones, representa un elemento de suma importancia, debido a que afecta al trabajador y su desempeño dentro de las mismas (Muñoz, Barrios y Cerón, 2017).

Asimismo, Trujillo, Valderrábano y Hernández (2007), refieren al mobbing como un elemento que afecta a la organización, dado a que repercute en la comunicación, en el desempeño de tareas en equipo, la eficiencia y clima organizacionales, lo que origina mayor rotación de personal, mayores costos y menor productividad en la empresa; por lo que, con base en lo anterior se puede inferir que, el problema de investigación se centra en la ausencia de equilibrio en las relaciones laborales dentro de las organizaciones. Dicho desequilibrio ocasiona estrés en los trabajadores, puesto que, no perciben congruencia entre el esfuerzo que realizan y las recompensas que obtienen por dicho esfuerzo (Cárdenas, Méndez \& González, 2014).

Por lo anterior, la presente investigación tiene el objetivo de identificar la existencia de dependencia estadística entre las dimensiones de mobbing y 
satisfacción laboral de los docentes de las IES usando la prueba de independencia estadística chi cuadrada.

\section{Mobbing y Satisfacción Laboral}

El mobbing tiene su origen en las ciencias naturales, particularmente en la etología (Trujillo et al., 2007); y, se deriva del verbo inglés "to mob", el cual generalmente se traduce como ataque, maltrato o asedio (Verona \& Santana, 2012b).

El mobbing es conocido de varias denominaciones, entre ellas: psicoterror, hostigamiento laboral, persecución encubierta, maltrato psicológico en el trabajo, violencia psíquica, acoso laboral y acoso moral (Verona \& Santana, 2012a). En la tabla 1 se presentan algunas definiciones con autores y año de conceptualización.

Tabla 1. Conceptos de mobbing

\begin{tabular}{|c|c|c|}
\hline Autor & Término & Definición \\
\hline Leymann (1996) & Mobbing & $\begin{array}{l}\text { Comportamiento o comunicación hostiles e } \\
\text { inmorales con extrema violencia psicológica, } \\
\text { realizados de forma sistemática y recurrente por uno } \\
\text { o varios individuos generalmente hacia un } \\
\text { individuo, con el objeto de destruir sus redes de } \\
\text { comunicación, reputación, perturbar el ejercicio de } \\
\text { su trabajo; lo anterior, con una frecuencia de al } \\
\text { menos una vez a la semana y con una duración de } \\
\text { por lo menos seis meses (Leymann, 1996:27). }\end{array}$ \\
\hline Hirigoyen (2001) & $\begin{array}{l}\text { Acoso moral/ } \\
\text { Mobbing }\end{array}$ & $\begin{array}{l}\text { Manifestación de conductas abusivas, que atenten } \\
\text { por su repetición o sistematización contra la } \\
\text { personalidad, dignidad o integridad física o psíquica } \\
\text { de la víctima, poniendo en peligro su empleo, o } \\
\text { degradando el ambiente de trabajo (Arciniega, } \\
\text { 2009). }\end{array}$ \\
\hline Romero (2006) & Mobbing & $\begin{array}{c}\text { Agresión, acoso, ataque y/o maltrato en masa o en } \\
\text { grupo a alguien (Vargas, 2011). }\end{array}$ \\
\hline Piñuel (2001) & Mobbing & $\begin{array}{l}\text { Maltrato verbal, modal e incluso cruel, continuo y } \\
\text { deliberado hacia un empleado por parte de otro u } \\
\text { otros, que tiene la finalidad de aniquilar o destruir al } \\
\text { trabajador psicológicamente y obtener su salida de } \\
\text { la organización (Piñuel \& Zabala, 2008:91). }\end{array}$ \\
\hline Arciniega (2009) & $\begin{array}{l}\text { Acoso moral/ } \\
\text { Mobbing }\end{array}$ & $\begin{array}{c}\text { Violencia psicológica en el trabajo con el objetivo } \\
\text { de intimidar, amedrentar, someter, doblegar o } \\
\text { deshacerse de la víctima. }\end{array}$ \\
\hline $\begin{array}{l}\text { Tengilimoğlu, D. } \\
\text { \& Mansur, F.A. } \\
\text { (2009) }\end{array}$ & Mobbing & $\begin{array}{l}\text { Comportamientos negativos, sistemáticos y } \\
\text { continuos contra un empleado por parte de uno o } \\
\text { más individuos en un ambiente de trabajo en el cual } \\
\text { se molesta a la víctima de manera física, psicológica } \\
\text { y social con el objetivo de que éste renuncie } \\
\text { (Tengilimoğlu \& Mansur, 2009:69). }\end{array}$ \\
\hline $\begin{array}{l}\text { Fernández y Nava } \\
\text { (2010) }\end{array}$ & $\begin{array}{l}\text { Mobbing/acoso moral } \\
\text { en el trabajo }\end{array}$ & $\begin{array}{l}\text { Conductas hostigadoras, agresivas, vejatorias y } \\
\text { humillantes efectuadas de forma sistemática, } \\
\text { reiterada y frecuente en el trabajo, dirigidas a } \\
\text { menoscabar la integridad psicológica de la víctima } \\
\text { con la finalidad de que salga de la organización. }\end{array}$ \\
\hline
\end{tabular}




\begin{tabular}{|c|c|c|}
\hline $\begin{array}{c}\text { Zabrodska, K. } \\
(2011)\end{array}$ & $\begin{array}{c}\text { Mobbing/ Acoso } \\
\text { moral }\end{array}$ & $\begin{array}{c}\text { Conductas agresivas y repetitivas en contra de una o } \\
\text { más personas que tienen dificultades para } \\
\text { defenderse. }\end{array}$ \\
\hline $\begin{array}{c}\text { Bustos, Caputo y } \\
\text { Aranda (2011) }\end{array}$ & $\begin{array}{c}\text { Hostigamiento } \\
\text { psicológico/ } \\
\text { moral laboral }\end{array}$ & $\begin{array}{c}\text { Violencia psicológica reiterada y por periodos } \\
\text { prolongados, ejercida por una o varias personas } \\
\text { hacia otra, para causar malestar y/o eliminar de la } \\
\text { organzación a la víctima (Bustos, Caputo, Aranda \& } \\
\text { Gimenez, 2015). }\end{array}$ \\
\hline $\begin{array}{c}\text { Moreno y Toro } \\
(2013)\end{array}$ & $\begin{array}{c}\text { Acoso } \\
\text { laboral/mobbing }\end{array}$ & $\begin{array}{c}\text { Acoso psicológico que denigra la dignidad humana } \\
\text { (Moreno \& Toro, 2013). }\end{array}$ \\
\hline
\end{tabular}

Elaboración propia, 2018.

Otro fenómeno de la vida laboral relacionado con las percepciones de los trabajadores respecto de sus empleos y que se refleja en su productividad, es la satisfacción laboral, definida por primera vez por Hoppock en 1935 (Civilidag, 2014). De acuerdo con Yew (2008) entre las definiciones más aceptadas se encuentra la de Locke que en 1969 la conceptualiza como un sentimiento emocional positivo, que resulta de la evaluación de lo que el empleado espera de su trabajo y lo que realmente obtiene; y, en 1976 refiere que es el resultado de las percepciones de su trabajo, valores y entorno.

En la tabla 2 se presentan algunas definiciones de satisfacción laboral y los autores de éstas.

Tabla 2. Conceptos de Satisfacción Laboral

\begin{tabular}{|c|c|c|}
\hline Autor & Término & Definición \\
\hline Hoppock, (1935) & Job Satisfaction & $\begin{array}{c}\text { "Combinación de eventos psicológicos físicos y } \\
\text { ambientales" (Civilidag, 2014:3) }\end{array}$ \\
\hline $\begin{array}{c}\text { Calvacante (2004), } \\
\text { Barraza y Ortega, } \\
(2009)\end{array}$ & Satisfacción Laboral & $\begin{array}{c}\text { Actitud generalizada respecto al empleo. Actitud } \\
\text { del trabajador frente a su trabajo (Barraza \& } \\
\text { Ortega, 2009). }\end{array}$ \\
\hline $\begin{array}{l}\text { Belkelman, (2004), } \\
\text { Dinham y Scott, } \\
\text { (2000) }\end{array}$ & Satisfacción Laboral & $\begin{array}{l}\text { Estado emocional del sentimiento que } \\
\text { experimentan los trabajadores con relación a sus } \\
\text { empleos. Grado en el que a los empleados les } \\
\text { gusta su trabajo (Anaya \& Suárez, 2010). }\end{array}$ \\
\hline $\begin{array}{c}\text { Morris y } \\
\text { Venkatesh (2010) }\end{array}$ & Satisfacción Laboral & $\begin{array}{l}\text { Favorabilidad o desfavorabilidad con la que las } \\
\text { personas evalúan su empleo, de acuerdo a } \\
\text { aspectos como salario, promoción y supervisión } \\
\text { (Omar, 2011). }\end{array}$ \\
\hline Madero (2012) & Satisfacción Laboral & $\begin{array}{l}\text { Actitud de la persona frente a su trabajo, respecto } \\
\text { a creencias, valores y características del puesto. } \\
\text { Indica la habilidad de la organización para } \\
\text { satisfacer sus necesidades (Madero, 2012) }\end{array}$ \\
\hline
\end{tabular}

Elaboración propia, 2018.

\section{Método}

El dominio del estudio se centra en cuatro instituciones de educación superior del Centro de México. Éstas fueron elegidas por representar un importante referente tanto en población, como en fuente de empleo en sus regiones. Otro factor considerado para la selección de las IES, fue la rotación de docentes que hace referencia el instrumento de medición, encontrándose 
mediante entrevistas dirigidas a miembros de las comunidades universitarias la existencia de una rotación de entre 54\% y 95\%, lo que las hizo elegibles para nuestro estudio.

Para el cálculo del tamaño de la muestra se empleó la fórmula para poblaciones finitas (menores de 10,000) (Murray \& Larry, 2009). La muestra estuvo constituida por 248 docentes, en ella se consideraron a los de tiempo completo $(20.16 \%)$ y por asignatura $(79.84 \%)$ de las cuatro IES, debido a que por su posición jerárquica pueden evaluar el mobbing vertical descendente y establecer su correlación con la satisfacción laboral. E1 57.26\% de los docentes son hombres y el $42.74 \%$ mujeres. De todos ellos, el $12.5 \%$ cuenta con estudios de doctorado, el 57.26\% de maestría y el $30.24 \%$ de licenciatura.

Para recolectar los datos de la investigación y medir las variables de estudio se diseñaron dos instrumentos; el primero de ellos, corresponde al mobbing, el cual fue desarrollado a partir del método CISNEROS (Cuestionario Interpersonal sobre Sicoterror, Ninguneo, Estigmatización y Rechazo en Organizaciones Sociales) de Iñaki Piñuel (Fidalgo \& Piñuel, 2004); y, en la escala NAQ-RE realizada por García-Izquierdo, Sáez, Ruiz, Blasco y Campillo (2004); y, para la satisfacción laboral se tomaron como base la Escala Multidimensional de Satisfacción Laboral Docente (EMSLD) (Barraza \& Ortega, 2009), y la Escala de Satisfacción Laboral-Versión para Profesores (ESL-VP) (Anaya \& Suárez, 2007), los cuales ya han sido validados en estudios realizados por los citados investigadores.

Se aplicó una prueba piloto a 30 docentes usando los instrumentos diseñados para verificar la confiabilidad de éstos. Se usó el método de consistencia interna basado en el Alfa de Cronbach, o dimensión teórica. Los valores obtenidos fueron de 0.97 para el instrumento de recolección de datos correspondiente a mobbing, y de 0.98 para el de satisfacción laboral.

Con la finalidad de analizar la relación de dependencia entre las dimensiones de mobbing (variable de respuesta) y las dimensiones de satisfacción laboral (variable explicativa) se usó la prueba de independencia estadística chi cuadrada, que es una prueba estadística utilizada para evaluar las hipótesis acerca de la relación entre dos variables categóricas (HernándezSampieri et al., 2010; Walpole, Myers \& Myers, 2012).

Las variables de interés en el presente estudio son el mobbing y la satisfacción laboral en IES, esto nos lleva a definir la siguiente:

Hipótesis de investigación. Existe una dependencia entre el mobbing y la satisfacción laboral de los docentes de las IES.

Tomando como referencia a Piñuel (2001 \& 2008) se consideraron como dimensiones de la variable mobbing: mobbing personal, desvalorización del trabajo, mobbing organizacional y sobrecarga de trabajo; y, para la variable satisfacción laboral se consideraron las dimensiones autorrealización, desempeño profesional, liderazgo y ambiente laboral, tomando como base a 
Barraza y Ortega (2009) y a Anaya y Suárez (2007). Dichas dimensiones fueron utilizadas para construir las hipótesis estadísticas.

\section{Resultados}

Con los datos recolectados de las cuatro IES, se contruyeron las tablas de contingencia o tabulación cruzada para probar cada hipótesis estadística. A continuación se presenta un resumen y discusión de los resultados obtenidos. Tabla 3. Tabla de contingencia para las variables mobbing personal y autorrealización con la satisfacción laboral de los docentes de las IES.

\begin{tabular}{cccc} 
& \multicolumn{2}{c}{ Autorrealización con la satisfacción laboral } & \\
\cline { 2 - 3 } Mobbing personal & $\mathrm{Si}$ & No & Total \\
\hline $\mathrm{Si}$ & 67 & 46 & 113 \\
$\mathrm{No}$ & 116 & 19 & 135 \\
\hline Total & 183 & 65 & 248 \\
\hline
\end{tabular}

\section{Hipótesis}

$\mathrm{H}_{0}$ : Las variables mobbing personal y autorrealización con la satisfacción laboral de los docentes de las IES son independientes.

$\mathrm{H}_{1}$ : Existe una dependencia entre las variables mobbing personal y autorrealización con la satisfacción laboral de los docentes de las IES.

Nivel de Significancia (alfa) $\alpha=0.05$

Estadístico utilizado: Prueba chi-cuadrada

Valor de $\mathrm{p}=4.126 \times 10^{-6} \quad \chi^{2}=21.205$, Grados de libertad $=1$

Toma de decisiones: Se rechaza la hipótesis nula, y se acepta la hipótesis alternativa.

Discusión: De acuerdo a la prueba estadística aplicada, existe una relación

estadísticamente significativa entre las variables analizadas. El valor de p, indica que dicha relación es fuerte, ya que está muy por debajo del nivel de significancia.

Tabla 4. Tabla de contingencia para las variables mobbing personal y satisfacción laboral con el desempeño profesional de los docentes de las IES.

Satisfacción laboral con el desempeño

profesional

\begin{tabular}{cccc}
\cline { 2 - 3 } Mobbing personal & $\mathrm{Si}$ & No & Total \\
\hline $\mathrm{Si}$ & 2 & 111 & 113 \\
$\mathrm{No}$ & 96 & 39 & 135 \\
\hline Total & 98 & 150 & 248 \\
\hline
\end{tabular}

\section{Hipótesis}

$\mathrm{H}_{0}$ Las variables mobbing personal y satisfacción laboral con el desempeño profesional de los docentes de las IES son independientes.

$\mathrm{H}_{1}$ : Existe una dependencia entre las variables mobbing personal y satisfacción laboral con el desempeño profesional de los docentes de las IES.

Nivel de Significancia (alfa) $\alpha=0.05$

Estadístico utilizado: Prueba chi-cuadrada

Valor de $\mathrm{p}=2.2 \times 10^{-10} \chi^{2}=120.86$, Grados de libertad $=1$

Toma de decisiones: La hipótesis nula se rechaza, y se acepta la hipótesis alternativa.

Discusión: Derivado de la aplicación de la prueba estadística, existe una relación estadísticamente significativa entre las variables analizadas. El valor de p indica que dicha relación es fuerte, debido a que está muy por debajo del nivel de significancia. 
Tabla 5. Tabla de contingencia para las variables mobbing personal y satisfacción laboral con el liderazgo de los docentes de las IES.

\begin{tabular}{cccc} 
& \multicolumn{2}{c}{ Satisfacción laboral con el liderazgo } & \\
\cline { 2 - 3 } Mobbing personal & $\mathrm{Si}$ & No & Total \\
\hline $\mathrm{Si}$ & 4 & 109 & 113 \\
$\mathrm{No}$ & 110 & 25 & 135 \\
\hline Total & 98 & 150 & 248 \\
\hline
\end{tabular}

\section{Hipótesis}

$\mathrm{H}_{0}$ Las variables mobbing personal y satisfacción laboral con el liderazgo de los docentes de las IES son independientes.

$\mathrm{H}_{1}$ : Existe una dependencia entre las variables mobbing personal y satisfacción laboral con el liderazgo de los docentes de las IES.

Nivel de Significancia (alfa) $\alpha=0.05$

Estadístico utilizado: Prueba chi-cuadrada

Valor de $\mathrm{p}=2.2 \times 10^{-16} \chi^{2}=147.33$, Grados de libertad $=1$

Toma de decisiones: Se rechaza la hipótesis nula, y se acepta la hipótesis alternativa.

Discusión: Observamos que existe una relación estadísticamente significativa entre las variables analizadas, dado que el valor de p está muy por debajo del nivel de significancia.

Tabla 6. Tabla de contingencia para las variables mobbing personal y satisfacción con el ambiente laboral de los docentes de las IES.

\begin{tabular}{cccc} 
& \multicolumn{2}{c}{ Satisfacción con el ambiente laboral } & \\
\cline { 2 - 3 } Mobbing personal & $\mathrm{Si}$ & No & Total \\
\hline $\mathrm{Si}$ & 32 & 81 & 113 \\
$\mathrm{No}$ & 81 & 54 & 135 \\
\hline Total & 113 & 135 & 248 \\
\hline
\end{tabular}

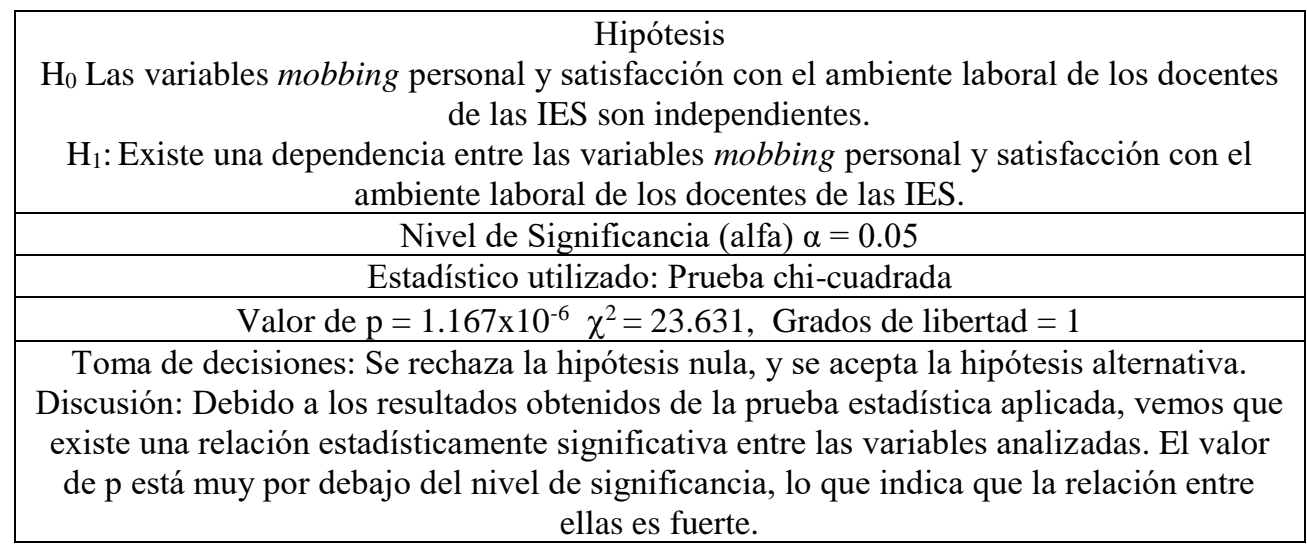


Tabla 7. Tabla de contingencia para las variables desvalorización del trabajo y autorrealización con la satisfacción laboral de los docentes de las IES.

\begin{tabular}{cccc} 
& \multicolumn{2}{c}{ Autorrealización con la satisfacción laboral } & \\
\cline { 2 - 3 } $\begin{array}{c}n \\
\text { Desvalorización del } \\
\text { trabajo }\end{array}$ & $\mathrm{Si}$ & No & Total \\
\hline $\mathrm{Si}$ & 74 & 52 & 126 \\
$\mathrm{No}$ & 109 & 13 & 122 \\
\hline Total & 183 & 65 & 248 \\
\hline
\end{tabular}

\section{Hipótesis}

$\mathrm{H}_{0}$ Las variables desvalorización del trabajo y autorrealización con la satisfacción laboral de los docentes de las IES son independientes.

$\mathrm{H}_{1}$ : Existe una dependencia entre las variables desvalorización del trabajo y autorrealización con la satisfacción laboral de los docentes de las IES.

$$
\text { Nivel de Significancia (alfa) } \alpha=0.05
$$

Estadístico utilizado: Prueba chi-cuadrada

Valor de $\mathrm{p}=1.167 \times 10^{-6} \chi^{2}=23.631$, Grados de libertad $=1$

Toma de decisiones: Se rechaza la hipótesis nula, y se acepta la hipótesis alternativa.

Discusión: De acuerdo a la prueba estadística aplicada, existe una relación

estadísticamente significativa entre las variables analizadas. El valor de $\mathrm{p}$, indica que

dicha relación es fuerte, ya que está muy por debajo del nivel de significancia.

Tabla 8. Tabla de contingencia para las variables desvalorización del trabajo y satisfacción laboral con el desempeño profesional de los docentes de las IES.

Satisfacción laboral con el desempeño

profesional

\begin{tabular}{cccc}
$\begin{array}{c}\text { Desvalorización del } \\
\text { trabajo }\end{array}$ & $\mathrm{Si}$ & No & Total \\
\cline { 2 - 3 } $\mathrm{Si}$ & 7 & 119 & 126 \\
$\mathrm{No}$ & 91 & 31 & 122 \\
\hline Total & 98 & 150 & 248 \\
\hline
\end{tabular}

\begin{tabular}{c} 
Hipótesis \\
$\mathrm{H}_{0}$ Las variables desvalorización del trabajo y satisfacción laboral con el desempeño \\
profesional de los docentes de las IES son independientes. \\
$\mathrm{H}_{1}$ : Existe una dependencia entre las variables desvalorización del trabajo y satisfacción \\
laboral con el desempeño profesional de los docentes de las IES. \\
\hline Nivel de Significancia (alfa) $\alpha=0.05$ \\
\hline Estadístico utilizado: Prueba chi-cuadrada \\
\hline Valor de $\mathrm{p}=2.2 \times 10^{-6} \chi^{2}=120.72$, Grados de libertad $=1$ \\
\hline $\begin{array}{c}\text { Toma de decisiones: Se rechaza la hipótesis nula, y se acepta la hipótesis alternativa. } \\
\text { Discusión: De acuerdo a la prueba estadística aplicada, el valor de p indica que existe una } \\
\text { relación fuerte, ya que está muy por debajo del nivel de significancia. }\end{array}$ \\
\hline
\end{tabular}


Tabla 9. Tabla de contingencia para las variables desvalorización del trabajo y satisfacción laboral con el liderazgo de los docentes de las IES.

Desvalorización del trabajo $\mathrm{Si}$ No Total

\begin{tabular}{ccc}
\hline \multicolumn{2}{c}{ Satisfacción laboral con el liderazgo } & \\
\cline { 1 - 2 } $\mathrm{Si}$ & No & Total \\
\hline 8 & 118 & 126 \\
106 & 16 & 122 \\
114 & 134 & 248 \\
\hline
\end{tabular}

\section{Hipótesis}

$\mathrm{H}_{0}$ Las variables desvalorización del trabajo y satisfacción laboral con el liderazgo de los docentes de las IES son independientes.

$\mathrm{H}_{1}$ : Existe una dependencia entre las variables desvalorización del trabajo y satisfacción laboral con el liderazgo de los docentes de las IES. Nivel de Significancia (alfa) $\alpha=0.05$ Estadístico utilizado: Prueba chi-cuadrada Valor de $\mathrm{p}=2.2 \times 10^{-16} \chi^{2}=158.64$, Grados de libertad $=1$

Toma de decisiones: Se rechaza la hipótesis nula, y se acepta la hipótesis alternativa. Discusión: El valor de p indica que existe una relación fuerte, ya que está muy por debajo del nivel de significancia.

Tabla 10. Tabla de contingencia para las variables desvalorización del trabajo y satisfacción con el ambiente laboral de los docentes de las IES.

\begin{tabular}{cccc} 
& \multicolumn{2}{c}{ Satisfacción con el ambiente laboral } & \\
\cline { 2 - 3 } $\begin{array}{c}\text { Desvalorización del } \\
\text { trabajo }\end{array}$ & $\mathrm{Si}$ & No & Total \\
\hline $\mathrm{Si}$ & 36 & 90 & 126 \\
$\mathrm{No}$ & 77 & 45 & 122 \\
\hline $\mathrm{Total}$ & 113 & 135 & 248 \\
\hline
\end{tabular}

\section{Hipótesis}

$\mathrm{H}_{0}$ Las variables desvalorización del trabajo y satisfacción con el ambiente laboral de los docentes de las IES son independientes.

$\mathrm{H}_{1}$ : Existe una dependencia entre las variables desvalorización del trabajo y satisfacción con el ambiente laboral de los docentes de las IES.

Nivel de Significancia (alfa) $\alpha=0.05$

Estadístico utilizado: Prueba chi-cuadrada

Valor de $\mathrm{p}=9.65 \times 10^{-8} \chi^{2}=28.443$, Grados de libertad $=1$

Toma de decisiones: Se rechaza la hipótesis nula, y se acepta la hipótesis alternativa.

Discusión: De acuerdo a la prueba estadística aplicada, existe una relación

estadísticamente significativa entre las variables analizadas. El valor de p indica que dicha relación es fuerte, ya que está muy por debajo del nivel de significancia. 
Tabla 11. Tabla de contingencia para las variables mobbing organizacional y autorrealización con la satisfacción laboral de los docentes de las IES.

Autorrealización con la

satisfacción laboral

\begin{tabular}{cccc} 
Mobbing organizacional & $\mathrm{Si}$ & No & Total \\
\hline $\mathrm{Si}$ & 68 & 47 & 115 \\
$\mathrm{No}$ & 115 & 18 & 133 \\
\hline Total & 183 & 65 & 248 \\
\hline
\end{tabular}

\section{Hipótesis}

$\mathrm{H}_{0}$ Las variables moobing organizacional y autorrealización con la satisfacción laboral de los docentes en las IES son independientes.

$\mathrm{H}_{1}$ : Existe una dependencia entre las variables moobing organizacional y autorrealización con la satisfacción laboral de los docentes en las IES.

Nivel de Significancia (alfa) $\alpha=0.05$

Estadístico utilizado: Prueba chi-cuadrada

Valor de $\mathrm{p}=2.172 \times 10^{-6} \quad \chi^{2}=22.436$, Grados de libertad $=1$

Toma de decisiones: La hipótesis nula se rechaza, y se acepta la hipótesis alternativa.

Discusión: Una vez realizada la prueba estadística, ésta indica que entre las variables analizadas existe una relación estadísticamente significativa, debido a que el valor de $\mathrm{p}$, muestra que dicha relación es fuerte, ya que está muy por debajo del nivel de significancia.

Tabla 12. Tabla de contingencia para las variables mobbing organizacional y satisfacción laboral con el desempeño profesional de los docentes de las IES.

\begin{tabular}{cccc}
\cline { 2 - 3 } & \multicolumn{2}{c}{$\begin{array}{c}\text { Satisfacción laboral con el desempeño } \\
\text { profesional }\end{array}$} & \\
\cline { 2 - 3 } $\begin{array}{c}\text { Mobbing } \\
\text { organizacional }\end{array}$ & $\mathrm{Si}$ & No & Total \\
\hline $\mathrm{Si}$ & 3 & 112 & 115 \\
$\mathrm{No}$ & 95 & 38 & 133 \\
\hline $\mathrm{Total}$ & 98 & 150 & 248 \\
\hline
\end{tabular}

\section{Hipótesis}

$\mathrm{H}_{0}$ Las variables mobbing organizacional y satisfacción laboral con el desempeño profesional de los docentes en las IES son independientes.

$\mathrm{H}_{1}$ : Existe una dependencia entre las variables mobbing organizacional y satisfacción laboral con el desempeño profesional de los docentes en las IES. Nivel de Significancia (alfa) $\alpha=0.05$ Estadístico utilizado: Prueba chi-cuadrada Valor de $\mathrm{p}=2.2 \times 10^{-16} \chi^{2}=119.35$, Grados de libertad $=1$

Toma de decisiones: No se acepta la hipótesis nula, por lo tanto se acepta la hipótesis alternativa.

Discusión: El resultado indica que existe una relación fuerte, pues el valor de p está muy por debajo del nivel de significancia. 
Tabla 13. Tabla de contingencia para las variables mobbing organizacional y satisfacción laboral con el liderazgo de los docentes de las IES.

Mobbing organizacional

\begin{tabular}{cccc}
\cline { 2 - 3 } $\begin{array}{c}\text { Mobbing } \\
\text { organizacional }\end{array}$ & \multicolumn{2}{c}{ Satisfacción laboral con el liderazgo } & \\
\cline { 2 - 3 } Total \\
\hline $\mathrm{Si}$ & $\mathrm{Si}$ & No & \\
\hline $\mathrm{No}$ & 5 & 110 & 115 \\
\hline Total & 109 & 24 & 135 \\
\hline
\end{tabular}

\begin{tabular}{c} 
Hipótesis \\
$\mathrm{H}_{0}$ Las variables mobbing organizacional y satisfacción laboral con el liderazgo de los \\
docentes en las IES son independientes. \\
$\mathrm{H}_{1}$ : Existe una dependencia entre las variables mobbing organizacional y satisfacción \\
laboral con el liderazgo de los docentes en las IES. \\
Nivel de Significancia (alfa) $\alpha=0.05$ \\
\hline Estadístico utilizado: Prueba chi-cuadrada \\
\hline $\begin{array}{c}\text { Toma de decisiones: Se rechaza la hipótesis nula, y se acepta la hipótesis alternativa. } \\
\text { Discusión: Derivado de la aplicación de la prueba estadística, se observa que existe } \\
\text { dependencia entre las variables analizadas, puesto que el valor de p está muy por debajo } \\
\text { del nivel de significancia. }\end{array}$ \\
\hline
\end{tabular}

Tabla 14. Tabla de contingencia para las variables mobbing organizacional y satisfacción con el ambiente laboral de los docentes de las IES.

\begin{tabular}{cccc} 
& \multicolumn{2}{c}{ Satisfacción con el ambiente laboral } & \\
\cline { 2 - 3 } $\begin{array}{c}\text { Mobbing } \\
\text { organizacional }\end{array}$ & $\mathrm{Si}$ & No & Total \\
\hline $\mathrm{Si}$ & & & 115 \\
$\mathrm{No}$ & 33 & 82 & 133 \\
\hline Total & 80 & 53 & 248 \\
\hline
\end{tabular}

\begin{tabular}{|c|}
\hline $\begin{array}{c}\text { Hipótesis } \\
\mathrm{H}_{0} \text { Las variables mobbing organizacional y satisfacción con el ambiente laboral de los } \\
\text { docentes en las IES son independientes. } \\
\mathrm{H}_{1} \text { : Existe una dependencia entre las variables mobbing organizacional y satisfacción con } \\
\text { el ambiente laboral de los docentes en las IES. }\end{array}$ \\
\hline Nivel de Significancia (alfa) $\alpha=0.05$ \\
\hline Estadístico utilizado: Prueba chi-cuadrada \\
\hline Valor de $\mathrm{p}=1.35 \times 10^{-6} \chi^{2}=23.35$, Grados de libertad $=1$ \\
\hline $\begin{array}{l}\text { Toma de decisiones: Se rechaza la hipótesis nula, y se acepta la hipótesis alternativa. } \\
\text { Discusión: Como resultado de la aplicación de la prueba estadística, se obtiene que existe } \\
\text { una relación estadísticamente significativa entre las variables analizadas. El valor de p, } \\
\text { indica que dicha relación es fuerte, ya que está muy por debajo del nivel de significancia. }\end{array}$ \\
\hline
\end{tabular}


Tabla 15. Tabla de contingencia para las variables sobrecarga de trabajo y autorrealización con la satisfacción laboral de los docentes de las IES.

\begin{tabular}{cccc}
\cline { 2 - 3 } $\begin{array}{c}\text { Sobrecarga de } \\
\text { trabajo }\end{array}$ & $\mathrm{Si}$ & Total \\
\cline { 2 - 3 } & & No & \\
\hline $\mathrm{Si}$ & 8 & 35 & 113 \\
$\mathrm{No}$ & 175 & 30 & 135 \\
\hline Total & 183 & 65 & 248 \\
\hline
\end{tabular}

\section{Hipótesis}

$\mathrm{H}_{0}$ : Las variables sobrecarga de trabajo y autorrealización con la satisfacción laboral de los docentes en las IES son independientes.

$\mathrm{H}_{1}$ : Existe una dependencia entre las variables sobrecarga de trabajo y autorrealización con la satisfacción laboral de los docentes en las IES.

Nivel de Significancia (alfa) $\alpha=0.05$

Estadístico utilizado: Prueba chi-cuadrada

Valor de $\mathrm{p}=2.2 \times 10^{-16} \chi^{2}=78.49$, Grados de libertad $=1$

Toma de decisiones: Se rechaza la hipótesis nula, y se acepta la hipótesis alternativa.

Discusión: La prueba estadística aplicada, muestra que existe una relación

estadísticamente significativa entre las variables analizadas, se obseva que dicha relación

es fuerte, debido a que el valor de p está muy por debajo del nivel de significancia.

Tabla 16. Tabla de contingencia para las variables sobrecarga de trabajo y satisfacción laboral con el desempeño profesional de los docentes de las IES.

\section{Satisfacción laboral con el desempeño} profesional

\begin{tabular}{cccc}
$\begin{array}{c}\text { Sobrecarga de } \\
\text { trabajo }\end{array}$ & Si & No & Total \\
\hline $\mathrm{Si}$ & 1 & 42 & 43 \\
$\mathrm{No}$ & 97 & 108 & 205 \\
\hline Total & 98 & 150 & 248 \\
\hline
\end{tabular}

\section{Hipótesis}

$\mathrm{H}_{0}$ Las variables sobrecarga de trabajo y satisfacción laboral con el desempeño profesional de los docentes en las IES son independientes.

$\mathrm{H}_{1}$ : Existe una dependencia entre las variables sobrecarga de trabajo y satisfacción laboral con el desempeño profesional de los docentes en las IES.

$$
\text { Nivel de Significancia (alfa) } \alpha=0.05
$$

Estadístico utilizado: Prueba chi-cuadrada

Valor de $\mathrm{p}=1.066 \times 10^{-7} \quad \chi^{2}=28.251$, Grados de libertad $=1$

Toma de decisiones: Se rechaza la hipótesis nula, y se acepta la hipótesis alternativa.

Discusión: Existe una relación estadísticamente significativa entre las variables analizadas. El valor de p, indica que dicha relación es fuerte, debido a que está muy por debajo del nivel de significancia. 
Tabla 17. Tabla de contingencia para las variables sobrecarga de trabajo y satisfacción laboral con el liderazgo de los docentes de las IES.

\begin{tabular}{cccc} 
& \multicolumn{1}{c}{ laboral con el liderazgo de los docentes de las IES. } & \\
\cline { 2 - 3 } $\begin{array}{c}\text { Sobrecarga de } \\
\text { trabajo }\end{array}$ & $\mathrm{Si}$ & No & Total \\
\cline { 2 - 3 } & & & \\
\hline $\mathrm{Si}$ & 0 & 43 & 43 \\
$\mathrm{No}$ & 114 & 91 & 205 \\
\hline Total & 114 & 134 & 248 \\
\hline
\end{tabular}

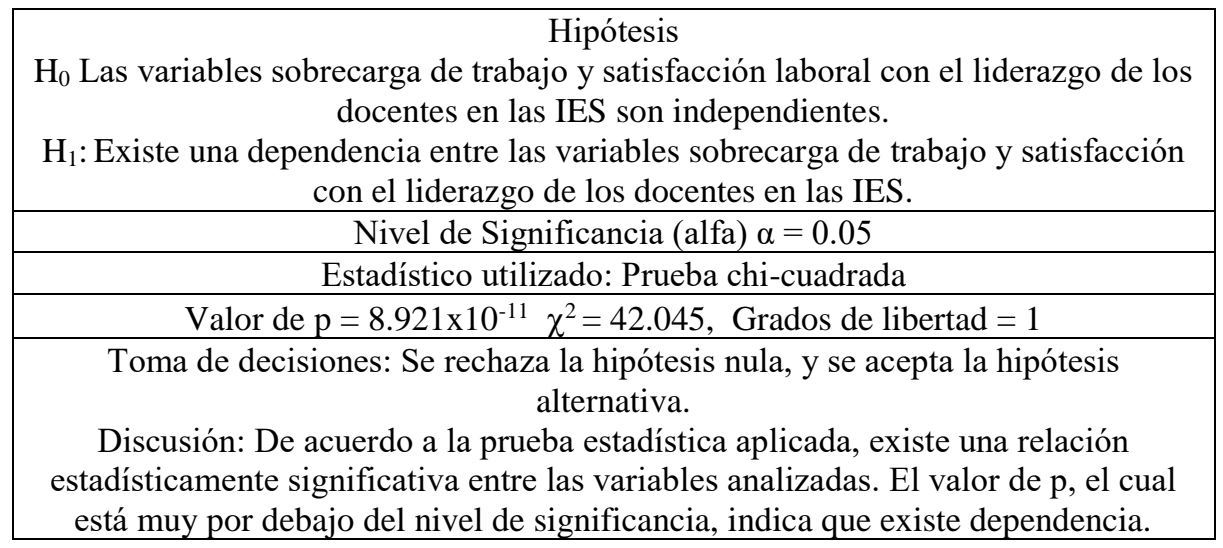

Tabla 18. Tabla de contingencia para las variables sobrecarga de trabajo y satisfacción con el ambiente laboral de los docentes de las IES.

\begin{tabular}{cccc} 
& \multicolumn{2}{c}{ Satisfacción con el ambiente laboral } & \\
\cline { 2 - 3 } $\begin{array}{c}\text { Sobrecarga de } \\
\text { trabajo }\end{array}$ & $\mathrm{Si}$ & No & Total \\
\hline $\mathrm{Si}$ & 0 & 43 & 43 \\
$\mathrm{No}$ & 113 & 92 & 205 \\
\hline Total & 113 & 135 & 248 \\
\hline
\end{tabular}

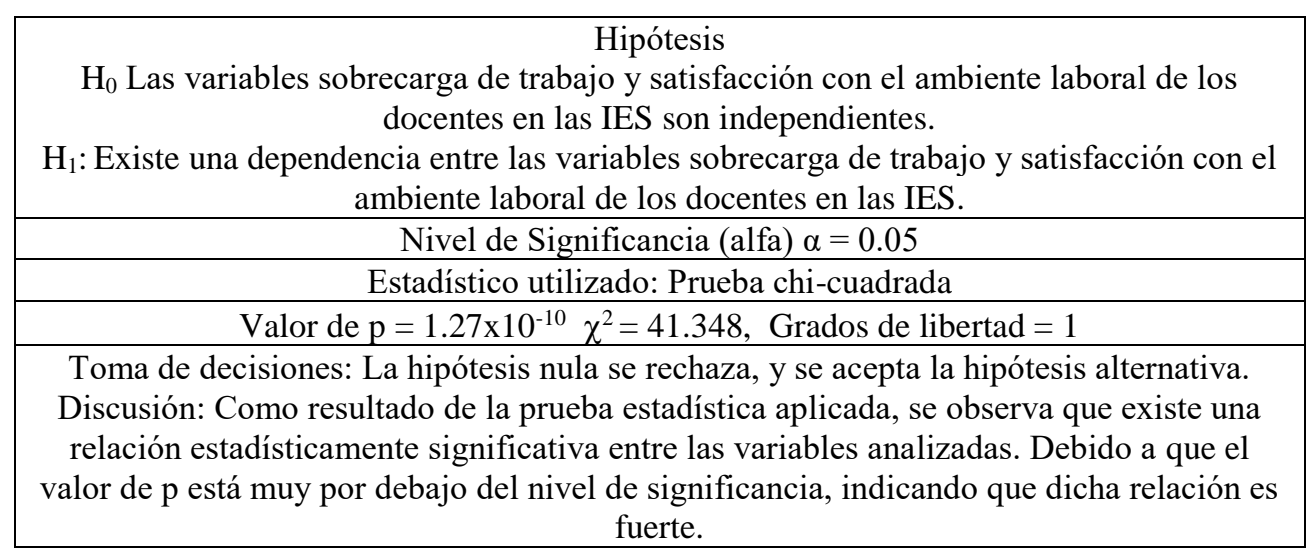




\section{Conclusion}

El mobbing es un elemento complejo que está presente en las organizaciones a partir de las relaciones interpersonales (Moreno-Jiménez, Rodríguez \& Garrosa, 2006; González-Trijueque \& Delgado, 2008), que convierte al espacio laboral en un lugar tóxico (Muñoz et al., (2017), por lo que los resultados obtenidos pueden ser de interés para los líderes y estudiosos de las empresas.

En la organización coexisten dos mundos, el material y el subjetivo (Pacheco, 2014), por ello, conocer la dependencia entre la satisfacción laboral y el mobbing resulta importante para comprender la vida simbólica en la organización. En esta investigación se realizaron pruebas estadíticas para conocer dicha dependencia entre las dimensiones de las variables mobbing y de satisfacción laboral.

Se comprobaron las hipótesis alternativas de la investigación, al encontrar que existe dependencia estadística significativa entre las dimensiones de mobbing y la satisfacción laboral de los docentes de las IES; es decir, se comprobó la existencia de dependencia entre las dimensiones mobbing personal, desvalorización del trabajo, mobbing organizacional y sobrecarga de trabajo, con las dimensiones de satisfacción laboral: autorrealización, desempeño profesional, liderazgo y ambiente laboral; para ello, se empleó la prueba de independencia estadística de chi-cuadrada. Los valores de $\mathrm{p}$ encontrados fueron de varios órdenes menor que el nivel de significancia elegido $(\alpha=0.05)$.

El mobbing representa una amplia gama de posibilidades de estudio no sólo en las IES, sino en todo tipo de entidades, con la presente investigación se espera contribuir a estudios posteriores que permitan la comprensión aún más profunda de este fenómeno y su relación con la satisfacción laboral, que hagan de la organización un lugar más humano y agradable para las personas y que permita su desarrollo integral.

Una vez comprobada la dependencia estadística entre las variables analizadas en este trabajo, se pueden proponer modelos predictivos, que permitan identificar la causalidad de los factores de las dimensiones de mobbing sobre la satisfacción laboral. Además, pueden analizarse cuáles de éstas son las que tienen mayor efecto.

\section{References:}

1. Anaya, N. D. \& Suárez, R. J. M. (2007). Satisfacción laboral de los profesores de educación infantil, primaria y secundaria. Un estudio de ámbito nacional. Revista de Educación, 344(), 217-243.

2. Anaya, N. D. \& Suárez, R. J. M. (2010). Evaluación de la satisfacción laboral del profesorado y aportaciones a su mejora en orden a la calidad 
de la educación. Revista Española de Orientación $y$ Psicopedagogía, 21(2), 283-294.

3. Arciniega, R. S. (2009). El acoso moral (mobbing) en las organizaciones laborales. Psicología Iberoamericana, 17(2), 13-23.

4. Arciniega, R. S. (2012). Factores organizacionales en la comprensión del mobbing. Psicología Iberoamericana, 20(1), 80-90.

5. Barraza, M.A. \& Ortega, M.F. (2009). Satisfacción laboral en instituciones formadoras de docentes. Un primer acercamiento. Revista electrónica de diálogos educativos, (17), 4-17.

6. Bustos, V. E., Caputo, M., Aranda, C. E. \& Gimenez, M. (2015). In V. E. Bustos, M. Caputo, C. E. Aranda, \& M. Gimenez (Eds.), Hostigamiento psicológico laboral e institucional en Iberoamérica: Estado del arte y experiencias de intervención (pp. 157-168). Argentina: Sb Editorial.

7. Calvacante, J. (2004). Satisfacción en el trabajo de los directores de escuelas secundarias públicas de la region de Jacobina (Bahía-Brasil). Tesis Doctoral de la Universidad Autónoma de Barcelona (on line).

8. Cárdenas, M., Méndez, L., \& González, M. (2014). Evaluación del desempeño docente, Estrés y Burnout en profesores universitarios. Revista Actualidades Investigativas Educación, 14(1), $1-22$.

9. Civilidag, A. (2014). Hotel employees mobbing, burnout, job satisfaction and perceived organizational support. A research on Hospitality in Turkey. European Scientific Journal, 10(35), 1-22.

10. Fernández, M. \& Nava, Y. (2010). El mobbing o acoso moral en el trabajo y su tratamiento en Venezuela. Revista de derecho: División de ciencias jurídicas de la Universidad del Norte. (33), 62-95.

11. Fidalgo, A. \& Piñuel, I. (2004). La escala Cisneros como herramienta de valoración del mobbing. Psicothema, 16(4), 615-624.

12. García Izquierdo-Izquierdo, M., Llor, B., García-Izquierdo, A. L. \& Ruiz, J. A. (2006). Bienestar psicológico en un amuestra de profesionales de los sectores educativo y sanotario (Psychological wellbeing and mobbing in a sample of professionals from the educational and health sectors). Revista de Psicología del trabajo y de las organizaciones, 22(3), 381-395.

13. González-Trijueque, D. \& Delgado, M. S. (2008). Acoso laboral y trnastornos de personalidad: un estudio con el MCMI-II. Clínica y salud, 19(2), 191-204.

14. Leymann, H. (1996). Mobbing. La persécution au travail. Editorial Du Seuil.

15. Locke, E.A. (1969). What is job satisfaction. Organizational Behaviour an Human Performance, 4(), 309-336. 
16. Locke, E.A. (1976). The Nature and causes of job satisfaction in M.D. Dunnette, Handbook of Industrial and Organizational Psychology, 1(), 1297-1349.

17. Madero, S. (2012). La efectividad de las compensaciones, la satisfacción del trabajador y las dimensiones del ambiente laboral. Ciencia-UANL, 15(57), 93-100.

18. Moreno-Jiménez, B., Rodríguez, A. y Garrosa, E. (2006). El papel moderador de la asertividad y la ansiedad social en el acoso psicológico en el trabajo: dos estudios empíricos. Revista de Psicología del Trabajo y de las Organizaciones, 22(3), 363-380.

19. Moreno, V.A.P. \& Toro, C.D.Y. (2013). Desprotección al acoso laboral. Nuevo derecho, 8(12), 107-115.

20. Morris, M. G., \& Venkatesh, V. (2010). Job characteristics and job satisfaction: understanding the role of enterprise resource planning system implementation. Mis Quarterly, 34(1), 143-161.

21. Muñoz, C.J.P., Barrios, Q.H. \& Cerón, I.A. (2017). Aproximación a la comprensión del mobbing como elemento que afecta a la organización. In ponencia presentada en el 5o Congreso Internacional de Investigación en Ciencias Económico Administrativas. San Agustín Tlaxiaca, Hidalgo. 1-14.

22. Omar, A. (2011). Liderazgo transformador y satisfacción laboral: el rol de la confianza en el supervisor. Liveravit, 17(2), 129-137.

23. Pacheco, A. (2014). La organización en búsqueda de sentido: producción material y producción simbólica en las organizaciones. $L a$ administración y los estudios organizacionales en el contexto latinoamericano, 1-16.

24. Piñuel, I. (2001). Mobbing: Cómo sobrevivir al acoso psicológico en el trabajo. Editorial Sal Terrae.

25. Piñuel, I. (2008). Mobbing, estado de la cuestión: todo lo que siempre quiso saber y nadie le explicó sobre el acoso psicológico en el trabajo. Barcelona, España: Gestión 2000.

26. Rodríguez, M.A., Retamal, P.M., Lizana, J. \& Cornejo, F. (2011). Clima y satisfacción laboral como predictores deldesempeño en una organización estatal chilena. Salud y sociedad, 2(2), 219-234.

27. Tengilimoğlu, D. \& Mansur, F.A. (2009). The effect of tehe mobbing (psychological violence) or organizational commitment in workplace. International Journal of Economic and Administratve Studies, 1(3), 69-84.

28. Trujillo, F. M., Valderrabano, A.M., \& Hernández, M. R. (2007). Mobbing: historia, causas, efectos y propuesta de un modelo para organizaciones mexicanas. Revista Innovar Journal Revista de Ciencias Administrativas y Sociales, 17(29), 71-91. 
29. Varela, O., Puhl, S.M. \& Izcurdia, M. (2013). Clima laboral y mobbing. Anuario de investigaciones, $X X(), 23-26$.

30. Vargas, T. J. A. (2011). Organización del trabajo y satisfacción laboral: un estudio de caso en la industria del calzado. Nova scientia, 4(7), 172-204.

31. Verona, M. \& Santana, R. (2012a). El mobbing: ¿el crimen perfecto? No, qué más quisieran "algunos". Criterio Libre, 10(17), 147-176.

32. Verona, M. \& Santana, R. (2012b). El mobbing: un problema ético en la gestión de empresas. Ciencia y sociedad. Ciencia y Sociedad, 37(4), 407-434.

33. Yew, L.T. (2008). Job satisfaction and affective commitment: a study of employees in the tourism industry in Sarawak, Malaysia. Sunway Academic Journal, (4), 27-43.

34. Zábrodská, K. (2011). Mobbing in higher education: an overview of international research. Ceskoslovenska Psychologie, 55(4), 332-344. 